\title{
Functional response: rigorous estimation and sensitivity to genetic variation in prey
}

\author{
Thomas Tully, Phillip Cassey and Régis Ferrière
}

Tully, T., Cassey, P. and Ferrière, R. 2005. Functional response: rigorous estimation and sensitivity to genetic variation in prey. - Oikos 111: 479-487.

\begin{abstract}
Holling's type II functional response is a cornerstone of community ecology and coevolutionary theory. The so-called disc equation is the most widely used model of the type II response, yet thus far no robust experimental assessment has been achieved in any single system. Fundamental issues that remain to be assessed include whether the assumptions of the disc equation are fulfilled, whether the disc equation yields accurate estimates of predation-related individual traits, and whether differences in disc equation parameters can capture genetic variation in prey behaviour. This paper provides a rigorous approach to all of these questions. The functional response of the predatory mite Pergamasus crassipes on three genetically distinct clones of the springtail Folsomia candida was measured at six levels of prey density in controlled conditions where prey number and arena size were concomitantly manipulated. A crucial assumption of Holling's disc equation was fulfilled by maintaining a constant prey density for the entire experimental period of predation. The timing of each attack and capture, as well as the duration of the handling time, were recorded by constant observation. We contrasted three different methods to calculate functional response curves: (1) indirect estimation of the disc equation's parameters from the number of prey killed by the end of each experimental run; (2) direct estimation of the parameters via a unique protocol of constant observation; and (3) independently deriving a function based on direct measurements of encounter rate and attack success. The basic assumptions of the disk equation were globally fulfilled. Estimations of the functional response's parameters (type II) were remarkably congruent across approach (1) and (2). A single genetic effect was detected - the relationship between the encounter rate and prey density differed significantly between clones - whereas a direct comparison of functional response across clones failed to reveal genetic variation.
\end{abstract}

T. Tully, P. Cassey and R. Ferrière, Lab. d'Écologie, CNRS UMR 7625, École Normale Supérieure, 46 rue d'Ulm, FR-75005 Paris, France (tully@biologie.ens.fr). PC also at: School of Biosciences, Univ. of Birmingham, Edgbaston, UK, B15 2TT. RF also at: Dept of Ecology and Evolutionary Biology, Univ. of Arizona, Tucson, AZ 85721, USA.

The number of prey that an individual predator kills is a function of prey density and is widely known as the 'functional response' (Holling 1966). Since the early work of Solomon (1949) and Holling (1959a, 1959b), functional responses have played a pivotal role in understanding predator-prey interactions and their ecological and evolutionary consequences on community dynamics. There are three basic curve types (type I, II and III), and many modifications (Jeschke et al. 2002), that have been used to model the functional response (Holling 1966). Type I models assume that the number of prey killed per predator increases linearly with increasing prey density until a maximum is reached. This model is often found to be biologically unrealistic, yet it remains fundamental to predator-prey coevolutionary theory. Most ecological interest in functional responses has involved model type II and III, in which saturation of the number of prey eaten occurs gradually in response to 
increasing prey density. Type III differs from type II in the assumption that the deceleration of the predation rate is preceded by an accelerating phase at low prey density. Type II functional responses have figured prominently in behavioural ecology, serving as the basis for foraging theory (Stephens and Krebs 1986, Abrams 1990). Similarly, mechanistic models of population dynamics of resource-limited consumers and predationlimited prey (Hassell 1978) have made extensive use of type II functional responses. In spite of several models predicting type III responses (Abrams 1982, 1987) and the theoretical demonstration of their dramatic consequences on the eco-evolutionary dynamics of predatorprey interactions (Dercole et al. 2002), type III responses remain poorly empirically tested.

Holling (1959a, 1959b) introduced the so-called disc equation as a general model of gradually saturating functional responses. The disc equation (drawing its name from a series of early experiments in which blindfolded humans 'preyed' on sandpaper discs) attributes the gradual saturation in the number of prey eaten per predator to the fact that the time available for searching for prey is progressively usurped by the time required to handle prey. The disc equation is given by:

$\mathrm{N}_{\mathrm{e}}=\frac{\mathrm{aNT}}{1+\mathrm{aNT}_{\mathrm{h}}}$

where $\mathrm{N}_{\mathrm{e}}$ is the number of prey eaten during the total exposure time $\mathrm{T}$, and $\mathrm{N}$ is prey density. The equation is parameterized by a, the instantaneous searching rate, and $T_{h}$, the mean handling time per prey (further details in Table 1). The disc equation and related functions have received wide acceptance as appealingly simple models of functional response in ecology and evolutionary biology (Hassel 1978, Crawley 1983, Williams and Juliano 1996).

The relationship between the disc equation and actual measurements of functional responses has been somewhat paradoxical (Abrams 1990). Although the disc equation often appears to provide an adequate fit for

Table 1. Model parameters and units.

\begin{tabular}{|c|c|}
\hline Parameter & Description and units \\
\hline $\mathrm{N}_{\mathrm{e}}$ & $\begin{array}{l}\text { Number of prey eaten during the time of the } \\
\text { experiment }\left(h^{-1}\right)\end{array}$ \\
\hline $\mathrm{N}$ & $\begin{array}{l}\text { Prey density (expressed in number of prey } 0.1123 \\
\mathrm{~m}^{-2} \text { ) }\end{array}$ \\
\hline $\mathrm{T}_{\mathrm{t}}$ & Total time of each experiment $(1 \mathrm{~h})$ \\
\hline $\mathrm{T}_{\mathrm{h}}$ & Handling time per prey item (h) \\
\hline $\mathrm{T}_{\mathrm{s}}$ & Time spent searching $(\mathrm{h})$ \\
\hline$a^{3}$ & $\begin{array}{l}\text { Instantaneous searching rate or attack constant } \\
\text { (probability of capturing a prey per unit of density } \\
\text { and time spent searching expressed in surface } \\
\text { cleared of prey per unit of time, } \mathrm{m}^{2} \mathrm{~h}^{-1} \text { ) }\end{array}$ \\
\hline e & $\begin{array}{l}\text { Encounter rate (number of prey encountered } \\
\text { detected and attacked, successfully or not, per unit } \\
\text { of time i.e. per hour in the experiment) }\end{array}$ \\
\hline$\sigma$ & $\begin{array}{l}\text { Attack success (probability of capturing a prey } \\
\text { which is being attacked). }\end{array}$ \\
\hline
\end{tabular}

functional response measurements, the values of a and $\mathrm{T}_{\mathrm{h}}$ as estimated from functional response curves seldom match those determined from direct behavioural observations. In this paper we suggest, and evaluate, possible reasons for these discrepancies. Notably, these reasons include: (i) data to which the disc equation is fitted are inappropriate, (ii) the biology of the predator-prey system violates basic assumptions of the disc equation model, (iii) fitting of the disc equation provides too little statistical power to yield accurate parameter estimates, (iv) disc equation parameter estimation is influenced by heterogeneity in the data generated e.g. by behavioural variation in prey or predators. The last issue also raises the question of whether the disc equation is appropriate for detecting variation in predation-related individual traits. This is a critical question when variation may be genetically based, and different prey or predator genotypes are being compared to determine which is the most effective at escaping a particular predator or killing a particular prey (Thompson 1975, Russo 1986, Kabissa et al. 1996, Nannini and Juliano 1998). For example, aphids have shown considerable genetic variation in escaping predators by producing varied winged morphs (Weisser et al. 1999, Braendle and Weisser 2001). Measuring such genetic variation in the functional response can enable comparisons of the evolutionary success of different genotypes in a given environment (Livdahl 1979, Houck and Strauss 1985, Juliano and Williams 1985, Abrams 2000, Yoshida et al. 2003), and predictions of how the comparisons would be affected by environmental change (Song and Heong 1997, Messina and Hanks 1998).

Hypothesis (i) is likely to be particularly relevant, given that the disc equation has been promoted, even in strictly inappropriate circumstances (Fan and Petitt 1994, 1997). These circumstances include the common practice of allowing prey density to deplete during exposure time to a given predator (reviewed by Juliano 2001). In fact, the disc equation makes no sense if prey density varies during the course of a predation episode (Abrams 1990). Nevertheless, this is commonly the case in most previous experiments, for which proper data analysis should involve fitting more complex equations (Rogers 1972, Juliano 2001). In the present paper we apply a rigorous experimental design where prey density is maintained as a constant in order to investigate how accurate parameter estimation is compared to direct measurements on individuals, and how sensitive the disc equation parameters are to genetic variation in prey. Moreover, we have also addressed the issue of whether prey number and patch size can affect functional response in addition to prey density. We used the mite Pergamasus crassipes (L., 1758) as a predator, and three genetically distinct clones of the springtail Folsomia candida (Willem, 1902) as prey. 


\section{Material and methods}

\section{Experimental procedure}

The experiments took place at the Biological Field Station of the École Normale Supérieure (Foljuif, Nemours, Seine-et-Marne, France). Adult predatory mites of the species Pergamasus crassipes were collected from leaf litter in the deciduous forest around the station one day prior to starting the experiment. The mites were individually kept in plastic rearing boxes $(2 \times 2 \times 2 \mathrm{~cm})$ and starved overnight to standardize their level of hunger. Each mite was used only once. Experiments were carried out with three clones (DK, GB and TO) of the blind and strictly parthenogenetic collembolan Folsomia candida which were chosen a priori for their dissimilar life history traits and different geographical origins (Tully 2004). Young adult collembola of equal size were used in all experiments in order to prevent simple effects of prey size/volume from influencing the form of the predation response. For each collembolan clone, prey were randomly selected from three replicated populations that were clonally descended from the same single female and had been maintained in laboratorystandardized conditions for several months.

Prey density was manipulated by varying both the number of prey per arena (1,2 or 4$)$ and the size of the arena $\left(70,140,280\right.$ or $\left.560 \mathrm{~cm}^{2}\right)$. This protocol provided six levels of surface density whose relative values are 1, 2, 4, 8, 16 and 32 (Table 2). For each treatment level (number of prey $\times$ surface of the arena) the experiment was replicated 24 times ( 8 times per clone), yielding a total of 288 trials. The arenas were made from $5 \mathrm{~cm}$ high circular plastic boxes with $1 \mathrm{~cm}$ thick plaster of Paris bases. The plaster base was smoothly surfaced with a thin black layer of clay, charcoal and ink which provided a good background contrast for behavioural observations. Both predators and prey remained exclusively on these experimental surfaces which were cleaned with a

Table 2. Four sizes of circular boxes were used as arenas in which one, two or four springtails were introduced. Prey density was expressed as number of prey per surface leading to six levels of density $(1,2,4,8,16$ and $32 ;$ a density of 1 corresponded to 18 collembola $\mathrm{m}^{-2}$, a density of 32 corresponded to 570 collembola $\mathrm{m}^{-2}$ ). For each level of number of prey and size of the arena 24 trials were conducted. The maximum number of prey a predator captured during one hour in our experiment is presented in brackets for each treatment level.

\begin{tabular}{lrrr}
\hline & \multicolumn{3}{c}{ Number of collembola introduced } \\
\cline { 2 - 4 } Surface $\mathrm{cm}^{2}$ (relative size) & \multicolumn{1}{c}{1} & 2 & 4 \\
\hline $70.2(1)$ & $8(5)$ & $16(7)$ & $32(8)$ \\
$140.4(2)$ & $4(4)$ & $8(6)$ & $16(8)$ \\
$280.8(4)$ & $2(4)$ & $4(3)$ & $8(7)$ \\
$561.5(8)$ & $1(2)$ & $2(1)$ & $4(4)$ \\
& \multicolumn{3}{c}{ Relative density } \\
\end{tabular}

wet brush after each trial. Experiments took place at room temperature $\left(\sim 20^{\circ} \mathrm{C}\right)$ and under constant light.

After the springtails were placed in an arena, a randomly chosen mite was introduced and its behaviour was monitored over a $1 \mathrm{~h}$ period. The successive times at which predatory events occurred were recorded; two types of predatory events were distinguished: an unsuccessful attack occurred when a mite encountered a springtail and unsuccessfully attempted to catch it; and a successful attack occurred when a mite successfully captured and killed a springtail. Handling times were also measured, as intervals between a successful attack and the time when the mite ceased eating or handling its prey item. In order to keep prey density constant during the course of the experiment, each time a collembolan was successfully attacked, a new one from the same clone was introduced to the arena. Results from a previous pilot experiment (not reported) enabled us to adjust both the levels of prey density and the duration $(1 \mathrm{~h})$ of the trials so that an encounter between prey or between a prey and a predator are sufficiently rare to avoid both the effect of interactions between prey or of predator satiation. In every treatment of our experiment, the mites were still searching for prey at the end of each trial. Although the vulnerability of a prey might have changed during the course of an experiment and varied between prey, monitoring the individual behaviour of the prey was beyond the logistical scope of this study.

\section{Statistical analysis}

All the analyses were conducted in version 1.8 of the software R (Ihaka and Gentleman 1996). Complex models were simplified by an automatic backward selection process (stepAIC function). Effects were tested by comparing nested models and using log likelihood ratio tests. Model parameters were estimated with restricted maximum likelihood methods (Pinheiro and Bates 2000). Additive effects of the number of prey, or size of the arena, with prey density were tested by comparing a model with density alone with a model with both density and number of prey, or density and size of the arena, as dependent variables.

\section{Functional response and disc equation fitting}

Functional response analysis raises the issue of distinguishing between type II and III. This can be done by fitting a quadratic curve on the proportion of prey eaten in relation to prey density. The sign of the slope near the origin (density $=0$ ) enables determining the type of the functional response (positive slope: type III function; negative slope: type II, Trexler et al. 1988, Juliano 2001). As most type III responses are expected to manifest only at very low prey densities (Hassell et al. 1977, Juliano 2001), we ensured, during a pilot experiment, that three 
of the six prey density treatments described above fell within the low-density range. For the lowest densities, the mean number of prey captures per mite was lower than one. Therefore, for these densities, the time spent handling prey was negligible compared to the time spent searching for prey.

Because our experimental design maintains, through replacement, a constant prey density, it is possible that a mite can eat more prey than the number of prey maintained in the arena at any one time. Therefore, the proportion of prey eaten was defined by choosing a virtual reference surface which preserved the relative values of prey densities tested but that was sufficiently large to ensure that the number of prey eaten by a predator never exceeded the expected number of prey present (Table 2). The reference surface was chosen as twice the largest arena surface, i.e. $2 \times 61.5 \mathrm{~cm}^{2}=0.1123$ $\mathrm{m}^{2}$. The corresponding relative densities $(2,4,8,16,32$ and 64) in Figs. 2 and 3 correspond successively to 17.8, 35.6, 71.2, 142.4, 284.9 and 569.8 prey $\mathrm{m}^{-2}$. A generalised mixed model (glmPQL function, link $=$ logit, $\mathrm{R}$ statistical package) was fitted to the binomial data (captured or not captured) in order to model this calibrated proportion with terms for clone and prey density, density squared, and density cubed as covariates and the code attributed to each mite as a random effect (Venable and Ripley 1999). Significance of continuous fixed variables were tested with t-tests and that of clone with a likelihood ratio test. The sign of the estimated parameters of the logistic regression was used to determine the type of the functional response. A nonlinear model was used to estimate the parameters a and $\mathrm{T}_{\mathrm{h}}$.

\section{Instantaneous searching rate, a}

Directly measuring parameter a raises the issue of separating bouts of searching versus prey handling. A simple way of directly estimating a is to use failure-time analysis (Haccou and Meelis 1992, Fox 2001) on data from the first successful attack for, by definition, prior to this event only searching time has been expended. In this case, the variable of interest is the time until an event (here, the successful attack of a prey) occurs; the corresponding 'rate of event' is known as the 'hazard rate'. For each level of density, the hazard rate $\mathrm{H}(\mathrm{N})$, that we assumed to be constant with time, was estimated directly by fitting a parametric (exponential) survival model to the time of first successful attack (survreg function in the Survival package of software R). The expected number of prey killed $\mathrm{N}_{\mathrm{e}}$ during the total searching time $T_{s}$ is $N_{e}=H(N) \times T_{s}$, and $a(N)$ can be estimated for each level of density as $\mathrm{a}(\mathrm{N})=\mathrm{H}(\mathrm{N}) / \mathrm{N}$. We used this approach to test for a dependency of a upon $\mathrm{N}$ and to provide estimates of the instantaneous searching rate.
Handling time, $T_{h}$

A direct estimate of $T_{h}$ was obtained by averaging handling times measured during the course of the experiments. We tested the hypothesis that $T_{h}$ is a constant parameter by analysing a linear mixed model of handling times that included clone and prey density as fixed effects and the mite code as a random effect (lme function in software R, Pinheiro and Bates 2000).

\section{Encounter rate, $e$}

The number of prey encountered and attacked, successfully or not, during $1 \mathrm{~h}$ was analysed with a general linear model with prey density and prey density squared as covariates (glm function for Poisson distributed data). Because the number of prey encountered is null when the density is zero, the model intercept was set to zero, and the effect of clone was tested as an interaction between prey density and clone.

\section{Attack success, $\sigma$}

Attack success (Table 1) was analyzed with a general linear mixed model (function glmmPQL) with a binomial variable (unsuccessful versus successful attack) as dependent variable, prey density and clone as fixed covariates, and the mite code as a random effect. Continuous fixed effects (density) were tested with t-tests and categorical effects (clone) with likelihood ratio tests.

\section{Results}

\section{(1) Functional response, and disc equation fitting}

We found no effect of clone $\left(\chi_{2}^{2}=0.108, \mathrm{P}=0.95\right)$, number of prey $\left(\mathrm{t}_{280}=-0.335, \mathrm{P}=0.73\right)$, nor box size $\left(t_{280}=0.478, P=0.63\right)$ on the proportion of prey eaten. Regarding prey density, neither cubic nor quadratic terms were significant $(\mathrm{P}>0.37)$, but the proportion of prey eaten decreased linearly with prey density (estimate $=-0.016, \mathrm{t}_{286}=-2.07, \mathrm{P}=0.04$ ) corresponding to a type II functional response. Fitting the disc equation to the data yielded $\mathrm{a}=0.167 \mathrm{~m}^{2} \mathrm{~h}^{-1}$, and $\mathrm{T}_{\mathrm{h}}=0.162 \mathrm{~h}=9 \mathrm{~min} 42 \mathrm{~s}$ (Fig. 1). The corresponding type-II functional response (for one hour, $\mathrm{T}=1$ ) reads (Fig. 2): $\mathrm{N}_{\mathrm{e}}=\frac{0.167 \times \mathrm{N}}{1+0.167 \times \mathrm{N} \times 0.162}$

\section{(2) Direct analysis of instantaneous searching rate, a, and handling time, $T_{h}$}

The analysis of time to first capture revealed that the hazard rate did not differ between clones $\left(\chi_{2}^{2}=0.78\right.$, $\mathrm{P}=0.67)$, nor depend on box size $\left(\chi_{1}^{2}=0.32, \mathrm{P}=0.568\right)$, but was affected by number of prey $\left(\chi_{1}^{2}=4.59, \mathrm{P}=0.032\right)$ 


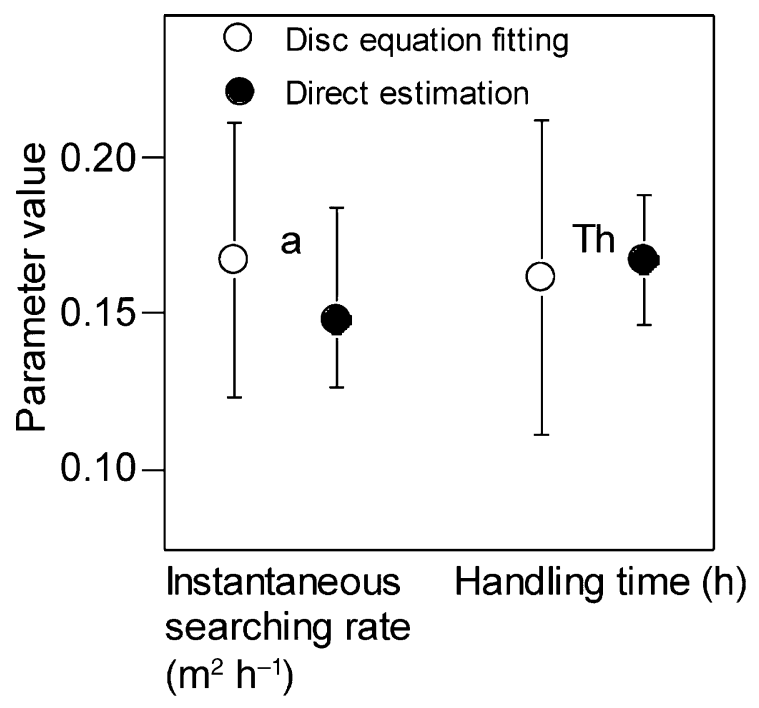

Fig. 1. Estimated values (with $95 \%$ confidence intervals) of the two parameters from the type II functional response, instantaneous searching rate a and handling time $\mathrm{T}_{\mathrm{h}}$, through disc equation fitting (method 1, see text for details) and direct measurements (method 2).

and prey density $\left(\chi_{1}^{2}=73.0, \mathrm{P}<0.01\right)$. The corresponding instantaneous searching rate did not increase with density $\left(\mathrm{F}_{1,4}=0.103, \mathrm{P}=0.76\right)$; its estimated mean value is $0.148 \mathrm{~m}^{2} \mathrm{~h}^{-1}$ (Fig. 1).

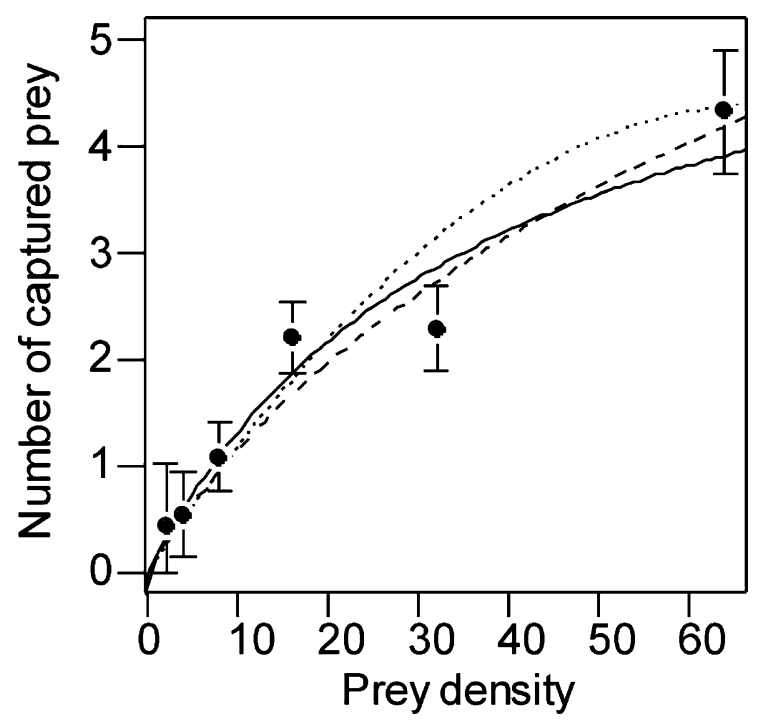

Fig. 2. Functional responses: data and models fitted through method 1-3. Mean number of prey captured (with 95\% confidence intervals) are represented for each level of prey density. Solid line: disc equation fitting (method 1). Dashed line: direct measurement of instantaneous searching rate and handling time inserted into the disc equation (method 2). Dotted line: product of the estimated encounter rate (quadratic in density, using mean estimate across clones for the slope parameter) with the estimated attack success (method 3).
Direct measurements showed that handling time was highly variable, varying from $10 \mathrm{~s}$ up to $28 \mathrm{~min}$. Handling time did not differ between the clones $\left(\chi_{2}^{2}=0.44, \mathrm{P}=0.80\right)$, nor did it depend on number of prey present in the box $\left(\chi_{1}^{2}=0.69, \mathrm{P}=0.40\right)$ or on box size $\left(\chi_{1}^{2}=0.17, \mathrm{P}=0.67\right)$. Importantly, handling time was not, as has been previously assumed, independent of density. At high density, the predators handled their prey for less time than at lower prey densities (Fig. 3A, $\mathrm{T}_{\mathrm{h}}$ (in seconds) $\left.=960-116 \times \log (\mathrm{N}), \chi_{1}^{2}=16.8, \mathrm{P}<0.01\right)$. However, this effect is mainly due to higher handling time at the lowest density, while it disappears for larger densities $\left(\chi_{1}^{2}=1.97, \mathrm{P}=0.16\right)$. The mean handling time estimated across all densities is $0.167 \mathrm{~h}$ or $10 \mathrm{~min} 1 \mathrm{~s}$ (Fig. 1). Inserting this estimate of $T_{h}$ and that of a into the disc equation gives the following functional response (Fig. 2): $\mathrm{N}_{\mathrm{e}}=\frac{0.148 \times \mathrm{N}}{1+0.148 \times \mathrm{N} \times 0.167}$

\section{(3) Encounter rate, e, and attack success, $\sigma$}

The encounter rate (number of encounters i.e. of successful and unsuccessful attacks per hour) increased with prey density (estimate $=0.250,95 \% \mathrm{CI}=[0.227 ; 0.274]$, $\left.\chi_{1}^{2}=905, \quad \mathrm{P}<0.01\right) \quad$ and decreased with squared prey density (estimate $=-0.0018,95 \% \mathrm{CI}=[-0.0023$; $\left.-0.0013], \chi_{1}^{2}=45.6, \mathrm{P}<0.01\right)$. The relationship between the encounter rate and density, as measured by the slope of its linear term, differed significantly between the clones $\left(\chi_{2}^{2}=6.74, P=0.03\right.$, Fig. 3B $)$. Pairwise comparisons of the clones revealed that DK and GB did not differ $\left(\chi_{1}^{2}=0.58\right.$, $\mathrm{P}=0.45)$, whereas TO was found to be more vulnerable than $\mathrm{DK}\left(\chi_{1}^{2}=6.73, \mathrm{P}=0.01\right)$ but not significantly more than $\mathrm{GB}\left(\chi_{1}^{2}=3.25, \mathrm{P}=0.07\right)$. When differences between clones are not taken into account, the encounter rate is modelled as: $\mathrm{e}(\mathrm{N})=0.2503 \times \mathrm{N}-0.0018 \times \mathrm{N}^{2}$.

Attack success did not differ between the clones $\left(\chi_{2}^{2}=2.41, P=0.29\right)$, and did not depend on number of prey $\left(\mathrm{t}_{220}=0.68, \mathrm{P}=0.49\right)$, box size $\left(\mathrm{t}_{220}=-0.13\right.$, $\mathrm{P}=0.89)$ or prey density $\left(\mathrm{t}_{221}=-1.47, \mathrm{P}=0.15\right)$. The mean estimate is $\sigma=0.514(95 \% \mathrm{CI}=[0.461 ; 0.566])$. Combining encounter rate (averaged across clones) and attack success yields a third model for the functional response (Fig. 2): $\mathrm{N}_{\mathrm{e}}=\mathrm{e} \times \sigma=(0.2503 \times \mathrm{N}-0.0018 \times$ $\left.\mathrm{N}^{2}\right) \times 0.514$.

\section{Discussion}

We were able to estimate the functional response of our collembola-mite system through three different approaches. The first two approaches involve two parameters, instantaneous searching rate and handling time, that are estimated either (1) by fitting the disc equation to the data or (2) by direct measurement of these parameters. 

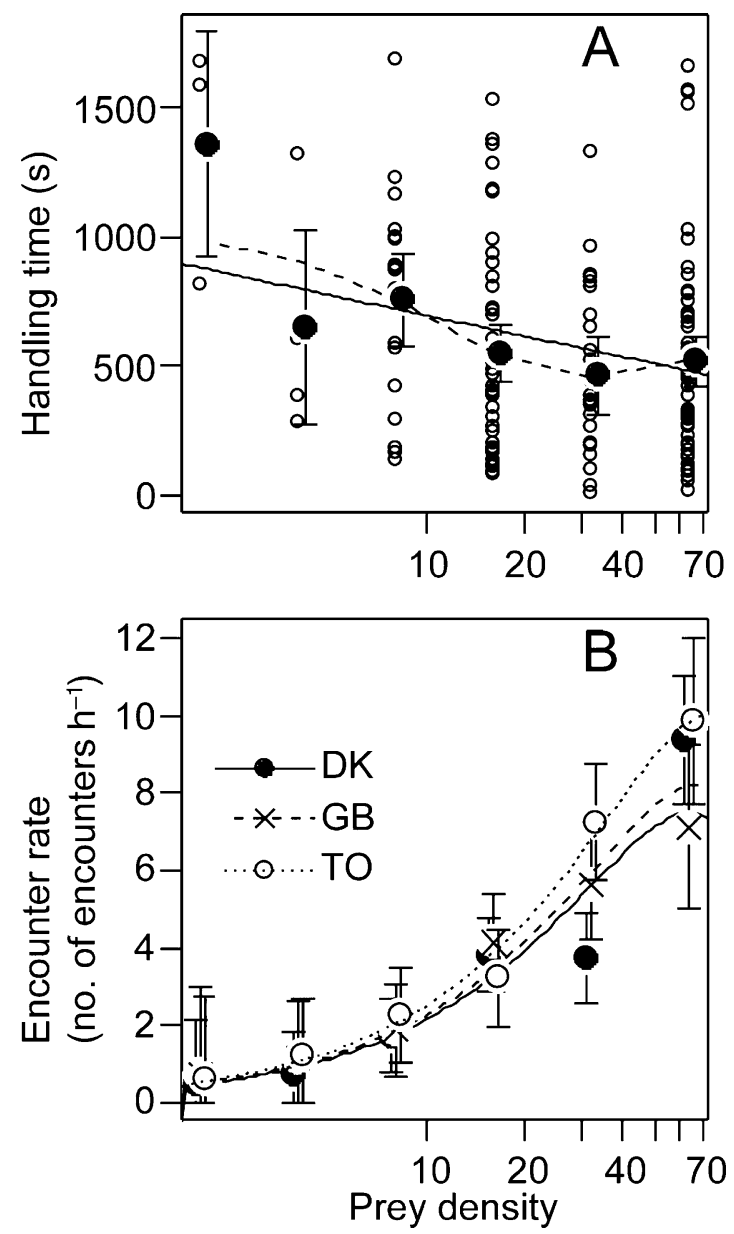

Fig. 3. (A) Handling time as a function of density (log scale). Raw data and mean handling time (with 95\% confidence intervals) are plotted for each level of prey density. Solid line: predicted response from a linear model fitted to handling time measurements. Dashed line: smoothing spline fitted to the raw data. (B) Mean number of prey encounters per hour (encounter rate) for each clone (DK, GB and TO) and level of density (with $95 \%$ confidence intervals) fitted by a quadratic equation (model structure: $\mathrm{N}_{\mathrm{e}} \sim$ clone $\times \mathrm{N}+\mathrm{N}^{2}$ ). The genetic variation on the slope of the linear term is illustrated by the difference in the three curves (one for each clone) predicted by the model.

The third approach (3) relates the number of prey eaten to the encounter rate and attack success. We ensured that our protocol enabled us to simultaneously (1) describe the functional response's type; (2) test the basic assumptions of the disk equation; (3) search for potential additive effects of arena size or prey number on prey density on the processes of predation and (4) look for genetic differences in prey susceptibility to predation.

\section{Type of functional response}

The type of functional response was determined both through fitting the disc equation and direct analysis of instantaneous searching rate. Both approaches failed to reveal any dependency of instantaneous searching rate with density. This was despite the experiment being conducted with several very low prey densities, at a region where the expected differences between a type II and a type III response are supposed to be greatest (Juliano 2001). Our rigorous protocol coupled with the consistency of both approaches confirmed that a type II functional response best described our particular system. This is indeed the most commonly observed form of functional response in laboratory experiments (Hassell 1978). It also demonstrated that the mites were not able to adjust their searching rate according to prey density. However, at low density, because the dependent variable is a proportion, the relative variability of this variable was greater than at higher densities where the number of measured events was higher. This implies that the quality of our estimators is lower at low densities (larger confidence intervals, Fig. 1) and may have reduced our power to detect an underlying type III response. An even larger number of replicates at lower densities would help counterbalance this effect (Juliano 2001). Because in this experiment, the functional response has been studied at relatively low prey densities and on a short time scale, the saturation of the functional response is mainly caused by direct handling of the prey rather than by digestion or satiation. Further investigations are needed to study, through conducting longer experiments, how the digestion process may affect the estimation of the handling time parameter and, consequently, the type and shape of the functional response.

\section{Basic assumptions}

The disc equation assumes that: (1) the total time spent foraging and the capture rate constant a are independent of prey density; (2) the time spent handling a given prey item is independent of prey density; and (3) the frequency of unsuccessful attacks on prey is independent of prey density. Abrams (1990) argued that each of these assumptions is likely to be violated if species behave adaptively, and gave examples of the functional response forms that may result from such violations.

Instantaneous searching rate was not only found to be independent of density but its direct measured value is very much similar to the one derived from fitting the disc equation (Fig. 1). Not surprisingly, estimates obtained from direct measurements (2), as opposed to fitting the disc equation (1), yielded narrower confidence intervals.

The second basic assumption, i.e. that handling time does not change with prey density, was partly violated. At low density, mites took more time to handle their prey (Fig. 3A). But this effect was apparently due to a specific response of handling time at the lowest density. In fact, we found handling time to be very variable. This 
can reflect strong hidden heterogeneity in the predator's behaviour and/or in variable qualities of the prey. Notably, it reinforces the idea that for sucking predators like mites, handling time is a highly flexible trait (Sih 1980). In our experiments we observed (but did not systematically record) that mites generally remained stationary while processing a prey but in some cases, the predator was able to continue searching in the arena while still sucking a captured collembola. In a few rare cases we even observed that a mite could capture a second prey without letting its first prey slip from its mandibles. Usually, however, handling was suddenly broken off if and when the predator encountered a second prey and attempted to capture it. These observations indicate that duration of handling can be partly determined by external environmental conditions such as prey density. Importantly, this observed flexibility in handling time may be an adaptive behaviour (Abrams 1990). Because encounter probability depends on prey density, an interruption of handling by a new encounter between a predator with a prey is more likely to happen when prey density is high. This kind of effect could explain the detected decrease of mean handling time with prey density. Despite the fact that measured handling times seem to be dependent on environmental conditions, it is remarkable that the mean observed value is essentially equal to the one indirectly estimated by fitting the disc equation (Fig. 1). In a stinkbug-potato beetle predator-prey experiment, Heimpel and Hough (1994) similarly found good agreement between estimated and observed values of handling time but not for searching rate. The good agreement that we found among our independent parameter estimates could be due to the fact that (1) contrary to most experiments we have rigorously kept prey density constant during the experiments and thus provided a rigorous estimation of our parameters when fitting the disk equation to our data (Juliano 2001), and (2) we have worked in conditions (prey densities and duration) where the mites did not get satiated during the trials. Therefore, our direct measurement of handling time is likely to better reflect the estimated handling time from the disk equation which is supposed to include time for attack, eating and digesting the prey (Jeschke et al. 2002). Again we emphasize that satiation and learning-which were insignificant in this study-might become substantial influences of the functional response over longer time scales or at higher densities.

Finally, we found the last assumption, that attack success is independent of density, to be fulfilled. Whatever the density, a mite had approximately a $50 \%$ chance of capturing a prey while chasing one. This intermediate value (Vermeij 1982) probably results from the coevolution of the jumping response time of the springtail with the speed of prey capture mechanism in the mite (Hopkin 1997). In our system an attack was nearly instantaneous. Thus, unsuccessful attacks do not significantly alter the time the mite spends searching for prey.

\section{Prey number and arena size}

Our protocol used identical prey densities in predation arenas of various sizes. We were able to provide a unique assessment of the potential for arena size and prey number to influence predation independently of prey density per se. When prey density is taken into account, neither arena size nor prey number influenced the variables that we have analyzed. We conclude that in our system it is the density of prey that mainly affects the predator-prey interaction. On the contrary, Wellenreuther et al. (2002) have shown that the magpie morwong coral fish is able to adjust it's predatory behaviour in response to both prey density and patch size. Such complex adaptive behaviour probably relies on visual cues which are lacking in our protagonists.

\section{Predator-prey behaviour}

Being blind, $F$. candida is not able to visually detect and avoid a predator. Although it has been shown that collembola can use predator or conspecific odour cues to adapt their movement, behaviour and spatial distribution (Nilsson and Bengtsson 2004), our regular cleaning of the surface of the arena before each trial probably helped avoid such behavioural responses in these experiments. Moreover, according to our continuous observations, neither the prey nor the predators seemed to be able to modify their seemingly 'random walk' when approaching each other. Regarding prey behaviour, the only defence mechanism that we observed was their ability to jump away with their furca after a mite attack. If the springtail managed to escape ( $\sim 50 \%$ of the times), the predators started to hunt more actively staying for a while in the close neighbourhood of the unsuccessful attack. These observations show that this system behaves closely to that of the experiment initially described by Holling (1959b).

\section{Genetic variation}

We found that the three clones differed in their vulnerability to predation, TO being more susceptible to being attacked than the other clones (Fig. 3B). Such a difference could, in fact, be due to a genetic difference in handling time and/or in level of prey activity. If a predator spent less time, on average, handling TO prey, then more time would be left for searching, hence leading to a larger number of encounters. However, because there is no clone effect of handling time, this 
interpretation is not supported. Nevertheless, a genetic difference in handling could result for instance from a genetic difference in growth rate and/or of adult body size. Indeed there is some genetic variation in these parameters (Tully 2004) but by standardizing the body size of the prey across clones we prevented the body size from directly affecting the form of the predation response. Instead we suggest that the observed difference might be due to different levels of prey activity and their subsequent interaction with a stationary predator. When the mite is motionless, the encounter rate will be proportional to the level of activity of the springtails. The higher vulnerability of the clone TO might therefore be due to the higher activity of this clone - a hypothesis that warrants further investigation.

Surprisingly, no clone effect was apparent between levels of the functional response itself. Similarly, differences in prey escape ability did not affect functional response in a polychaete-amphipod predator-prey system (Abrams et al. 1990). This could result from other compensating genetic effects. Indeed, albeit not significant, the attack success of the clone TO is lower than the one of the other two clones. Subsequently, both of these effects (encounter rate and attack success) tend to compensate and could explain why no genetic differences are detected between the number of prey captured. More detailed work are needed to confirm and eventually clarify these mechanisms. The negative effect of density square on the encounter rate emphasizes the fact that, at high density, the mites spend on total more time handling their prey and therefore less time searching.

\section{Conclusion}

Individual traits that affect the ability to accomplish or avoid predation have major effects on fitness and should be under strong selection. However, the evolution of traits related to predation in both predator and prey has proven to be difficult to understand in theory, and difficult to study empirically. These difficulties have not prevented the growth of theory predicting the potential evolutionary trajectories of predator-prey interactions. However, so far the theory has largely remained untested (Abrams 2000). One way to make a connection between theory and observations from real systems is to demonstrate that key assumptions of the models are satisfied. Essential to evolutionary modelling are assumptions about how adaptive traits relate to model parameters, and how these parameters influence, and how they are influenced by, population densities. Predator-prey coevolutionary models have assumed that (1) genetic variation in underlying adaptive traits translates into variation in these parameters, and (2) both parameters are independent of prey density. We found that clonal variation in prey vulnerability (encounter rate) exists, but this variation is not detected when functional response curves are fitted to the data. Nevertheless, due to sensitivity of model predictions to even tiny changes in their specification (Wood and Thomas 1999), we recognise that the long term evolutionary consequences of such genetic variation should still be considered in further coevolutionary models. Also, density affects handling time negatively - an effect which is not reflected in Holling's functional responses. Such results urge coevolutionary theories to incorporate more detailed models of prey and predator behaviour if they are to yield predictions on the adaptive significance of trait variation to be empirically tested.

Acknowledgements - We are grateful to Nathan Pike for his help during the experiment and for helpful comments on an earlier version of the manuscript, to Christina Weidick Kærsgaard (Dept of Terrestrial Ecology, DMU, Soil Fauna and Ecotoxicology Research Unit, Silkeborg, Denmark), Anne Bedos and Louis Deharveng (Muséum d'Histoire Naturelle, Paris, France) for providing the clones of $F$. candida, and to Lars R. Lundqvist (Museum of Zoology, Lund Univ., Sweden) for identifying $P$. crassipes.

\section{References}

Abrams, P. A. 1982. Functional responses of optimal foragers. - Am. Nat. 120: 382-390.

Abrams, P. A. 1987. The nonlinearity of competitive effects in models of competition for essential resources. - Theor. Popul. Biol. 32: 50-65.

Abrams, P. A. 1990. The effects of adaptive behavior of the type-2 functional response. - Ecol. Washington DC 71: $877-885$

Abrams, P. A. 2000. The evolution of predator-prey interactions: theory and evidence. - Annu. Rev. Ecol. Syst. 31: 79105.

Abrams, P. A., Hill, C. and Elmgren, R. 1990. The functional response of the predatory polychaete Harmothoe sarsi to the amphipod Pontoporeia affinis. - Oikos 59: 261-269.

Braendle, C. and Weisser, W. W. 2001. Variation in escape behavior of red and green clones of the pea aphid. - J. Insect Behav. 14: 497-509.

Crawley, M. J. 1983. Herbivory: the dynamics of animal-plant interactions. - Univ. of California Press.

Dercole, F., Ferrière, R. and Rinaldi, S. 2002. Ecological bistability and evolutionary reversals under asymmetrical competition. - Evolution 56: 1081-1090.

Fan, Y. Q. and Petitt, F. L. 1994. Parameter estimation of the functional response. - Environ. Entomol. 23: 785-794.

Fan, Y. Q. and Petitt, F. L. 1997. Functional response, variance, and regression analysis: A reply to Williams and Juliano. - Environ. Entomol. 26: 1-3.

Fox, G. A. 2001. Failure-time analysis. - In: Scheiner, S. M. and Gurevitch, J. (eds), Design and analysis of ecological experiments. Oxford Univ. Press.

Haccou, P. and Meelis, E. 1992. Statistical analysis of behavioural data: an approach based on time-structured models. - Oxford Univ. Press.

Hassell, M. P. 1978. The dynamics of arthropod predator-prey systems. - Princeton Univ. Press.

Hassell, M. P., Lawton, J. H. and Beddington, J. R. 1977. Sigmoid functional responses by invertebrate predators and parasitoids. - J. Anim. Ecol. 46: 249-262.

Heimpel, G. E. and Hough, G. J. A. 1994. Components of the functional response of Perillus bioculatus (Hemiptera: Pentatomidae). - Environ. Entomol. 23: 855-859. 
Holling, C. S. 1959a. The components of predation as revealed by a study of small-mammal predation of the European pine sawfly. - Can. Entomol. 91: 293-320.

Holling, C. S. 1959b. Some characteristics of simple types of predation and parasitism. - Can. Entomol. 91: 385-398.

Holling, C. S. 1966. The functional response of invertebrate predators to prey density. - Mem. Entomol. Soc. Can. 48: $1-86$.

Hopkin, S. P. 1997. Biology of the springtails (Insecta: Collembola). - Oxford Univ. Press.

Houck, M. A. and Strauss, R. E. 1985. The comparative study of functional responses, experimental design and statistical interpretation. - Can. Entomol. 117: 617-630.

Ihaka, R. and Gentleman, R. 1996. R: a language for data analysis and graphics. - J. Comput. Graph. Stat. 5: 299314.

Jeschke, J. M., Kopp, M. and Tollrian, R. 2002. Predator functional responses: discriminating between handling and digesting prey. - Ecol. Monogr. 72: 95-112.

Juliano, S. A. 2001. Nonlinear curve fitting. - In: Scheiner, S. M. and Gurevitch, J. (eds), Design and analysis of ecological experiments. Oxford Univ. Press, pp. 178-198.

Juliano, S. A. and Williams, F. M. 1985. On the evolution of handling time. - Evolution 39: 212-215.

Kabissa, J. C. B., Yarro, J. G., Kayumbo, H. Y. et al. 1996. Functional responses of two chrysopid predators feeding on Helicoverpa armigera (Lep: Noctuidae) and Aphis gossypii (Hom: Aphididae). - Entomophaga 41: 141-151.

Livdahl, T. P. 1979. Evolution of handling time-functionalresponse of a predator to the density of sympatric and allopatric strains of prey. - Evolution 33: 765-768.

Messina, F. J. and Hanks, J. B. 1998. Host plant alters the shape of the functional response of an aphid predator (Coleoptera: Coccinellidae). - Environ. Entomol. 27: 1196-1202.

Nannini, M. A. and Juliano, S. A. 1998. Effects of the facultative predator Anopheles barberi on population performance of its prey Aedes triseriatus (Diptera Culicidae). - Ann. Entomol. Soc. Am. 91: 33-42.

Nilsson, E. and Bengtsson, G. 2004. Death odour changes movement pattern of a Collembola. - Oikos 104: 509-517.

Pinheiro, J. C. and Bates, D. M. 2000. Mixed-effects models in S and S-PLUS. - Springer-Verlag.

Rogers, D. J. 1972. Random search and insect population models. - J. Anim. Ecol. 41: 369-383.

Russo, R. 1986. Comparison of predatory behavior in five species of Toxorhynchites (Diptera: Culicidae). - Ann. Entomol. Soc. Am. 77: 312-318.
Sih, A. 1980. Optimal foraging-partial consumption of prey. - Am. Nat. 116: 281-290.

Solomon, M. E. 1949. The natural control of animal populations. - J. Anim. Ecol. 18: 1-35.

Song, Y. H. and Heong, K. L. 1997. Changes in searching responses with temperature of Cyrtorhinus lividipennis Reuter (Hemiptera: Miridae) on the eggs of the brown planthopper, Nilaparvata lugens (Stal.) (Homoptera: Delphacidae). - Res. Popul. Ecol. 39: 201-206.

Stephens, D. W. and Krebs, J. R. 1986. Foraging theory. - Princeton Univ. Press.

Thompson, D. J. 1975. Towards a predator-prey model incorporating age structure: the effects of predator and prey size on the predation of Daphnia magna by Ischnure elegans. - J. Anim. Ecol. 44: 907-916.

Trexler, J. C., McCulloch, C. E. and Travis, J. 1988. How can the functional response best be determined. - Oecologia 76: $206-214$

Tully, T. 2004. Facteurs maternels, génétiques et environnementaux de l'expression des traits d'histoire de vie chez le collembole Folsomia candida, Willem (Isotomidae). Ecole Doctorale Diversité du Vivant. Univ. Pierre et Marie Curie, p. 237.

Venable, W. N. and Ripley, B. D. 1999. Modern applied statistics with S-PLUS. - Springer-Verlag.

Vermeij, G. J. 1982. Unsuccessful predation and evolution. - Am. Nat. 120: 701-720.

Weisser, W. W., Braendle, C. and Minoretti, N. 1999. Predatorinduced morphological shift in the pea aphid. - Proc. R. Soc. Lond. Ser. B 266: 1175-1181.

Wellenreuther, M. and Connell, S. D. 2002. Response of predators to prey abundance: separating the effects of prey density and patch size. - J. Exp. Mar. Biol. Ecol. 273: 6171.

Willem, V. 1902. Note préliminaire sur les collemboles des grottes de Han et de Rochefort. - Ann. Soc. Entomol. Belg. 46: $275-283$.

Williams, F. M. and Juliano, S. A. 1996. Functional responses revisited. - Environ. Entomol. 25: 549-550.

Wood, S. N. and Thomas, M. B. 1999. Super-sensitivity to structure in biological models. - Proc. R. Soc. Lond. Ser. B 266: 565-570.

Yoshida, T., Urabe, J. and Elser, J. J. 2003. Assessment of 'topdown' and 'bottom-up' forces as determinants of rotifer distribution among lakes in Ontario, Canada. - Ecol. Res. 18: $639-650$. 